\title{
Molecular Analysis of Centrifugation Supernatant Fluid from Pancreaticobiliary Duct Samples Can Improve Cancer Detection
}

\author{
Sydney D. Finkelstein ${ }^{a} \quad$ Marluce Bibbo $^{b} \quad$ David E. Loren ${ }^{b} \quad$ Ali A. Siddiqui ${ }^{b}$ \\ Charalambos Solomides $^{b}$ Thomas E. Kowalski ${ }^{b}$ Eric Ellsworth ${ }^{a}$ \\ ${ }^{a}$ RedPath Integrated Pathology, Inc., Pittsburgh, Pa., and ${ }^{\text {b } T h o m a s ~ J e f f e r s o n ~ U n i v e r s i t y ~ H o s p i t a l, ~ P h i l a d e l p h i a, ~ P a ., ~ U S A ~}$
}

\section{Key Words}

Bile duct stricture - Cytocentrifugation - Genotyping •

KRAS - Loss of heterozygosity • Mutations - Pancreatic duct stricture $\cdot$ Supernatant

\begin{abstract}
Objective: We aimed to supplement microscopic examination of biliary cytobrush specimens to improve sensitivity by mutational profiling of: (1) selected cells microdissected from cytology slides and (2) corresponding cell-free DNA in residual supernatant fluid. Study Design: From 43 patients with brushings of bile or pancreatic duct strictures, DNA was extracted from microdissected cells and 1-2 $\mathrm{ml}$ of cytocentrifugation supernatant fluid. Mutational analysis targeted 17 genomic sites associated with pancreaticobiliary cancer, including sequencing for KRAS point mutation and loss of heterozygosity $(\mathrm{LOH})$ analysis of microsatellites located at 1p, 3p, 5q, 9p, 10q, 17p, 17q, 21q, and 22q. Results: Mutations were found in $25 / 28$ patients with malignancy, and no mutations were found in $5 / 5$ patients with benign surgical results. The cell-free supernatant fluid generally contained higher levels and quality of DNA, resulting in increased detection of mutations in most patients. KRAS mutations only occurred in patients with pancreatic cancer. Mutational profiling of supernatant fluid specimens resulted in high sensitivity and specificity for malignancy, improving the detection of malignancy over cytology alone. Conclusion: Brush cytology
\end{abstract}

specimens yielded supernatant fluid enriched with DNA, probably from actively proliferating cells. Mutational profiling can enhance the cytologic evaluation and characterization of specimens suspected to contain pancreatic or bile duct cancer.

Copyright $\odot 2012$ S. Karger AG, Basel

\section{Introduction}

Microscopic examination of pancreatic and bile duct brushing samples ranks among the most challenging areas of cytology practice. Inflammatory states can produce significant reactive cellular atypia which, further modified by cellular degeneration effects, overlaps features seen in neoplastic strictures. Similarly, the lack of an adequate number of intact representative cells in a brushing specimen may prevent accurate diagnosis. Both phenomena together make diagnosis difficult and limit diagnostic sensitivity for pancreatic and biliary cancer. While recent advances in endoscopic visualization (e.g. confocal microscopy) and sampling have improved sensitivity for cancer $[1,2]$, it continues to remain suboptimal, ranging from $25-50 \%[3,4]$. Diagnostic modalities that improve both detection and exclusion of cancer requiring high positive and negative predictive values would aid in resolving this dilemma and lead to better management of patients.

Correspondence to: Dr. Sydney Finkelstein 
Because actively developing neoplasms are fundamentally characterized by increased cell turnover and progressive mutation acquisition, the cellular environment in the region of such a neoplasm often contains free DNA shed from neoplastic cells [5]. While such shedding of cells and cellular DNA is often discussed for carcinomas within a duct, we hypothesized that a cancer outside of the lumen of a strictured duct could also lead to high levels of mutated DNA within the duct. We based this hypothesis on extensive work showing that free circulating DNA in serum and plasma has demonstrated that DNA from tumors and other cells undergoing active proliferation can end up well outside of the organ [5]. When such free DNA is from a neoplasm, it will contain the mutations responsible for neoplastic progression of the proliferating cells. Conversely, active inflammatory processes can also generate shed cells and associated free DNA, but since these are not related to neoplastic progression, this DNA will lack mutations. We further hypothesized that if this DNA leaks across the duct wall to the internal lining, then cytobrushing of this lining will pick up both cells from the duct itself and leached DNA. If the free DNA shed from the tumor could be isolated from other cells, we would therefore expect to see mutations in this DNA when it comes from a neoplastic process and no mutations in this DNA when the process is nonneoplastic.

To test this phenomenon in a clinical context, we asked whether cytobrushing specimens from strictured pancreatic or bile ducts surrounded by or adjacent to cancer would contain free DNA, and further whether mutations could be detected in this fluid. The supernatant fluid that is generated during the centrifugation of cytobrush cells for cytology slide preparation provided an excellent avenue to test these hypotheses.

\section{Materials and Methods}

\section{Initial Specimen Handling}

Under IRB approval, we retrieved stained cytology slides and corresponding residual supernatant fluid specimens from 43 patients with a pancreatic or biliary duct stricture. The overarching objective was to assess the clinical utility of ancillary mutation detection when first-line cytology microscopic analysis proved inadequate or indeterminate affording the opportunity for second-line molecular analysis to provide clinically actionable information.

Cytology adequacy assessment and diagnosis were carried out using standard morphologic criteria [6]. Direct smear slides were first prepared from the cytobrush, and then the brush was rinsed in 5-10 $\mathrm{ml}$ of Saccomanno's fixative and the rinsed sample underwent standard cytocentrifugation. The residual supernatant, maintained at $4^{\circ} \mathrm{C}$, was retained for DNA extraction and mutational analysis.

All cases received cytology assessment as shown in tables 1 and 2. Cellularity ranged from acellular to highly cellular, with most cases exhibiting cellularity lower than is desirable for morphologic interpretation. Thus, while cases with low cellularity were characterized via cytology to the best degree possible, the final cytologic diagnosis was often indeterminate/inadequate for clinical decision making. In our analysis, such cases were generally categorized as 'no significant atypia observed'.

\section{Study Population}

Two cohorts of patients were formulated as follows. The first cohort (shown in table 1) consisted of specimens from 18 patients with proven cancer determined via histopathology examination of a surgical resection specimen. Within this cohort, there was a range of cytology diagnoses (7 benign, 4 atypical, 5 suspicious, and 2 malignant), though the ultimate preoperative cytology diagnosis was frequently inadequate or indeterminate due to low cellularity. In the first cohort, we tested only stained cytology slides; cells from these slides were microdissected and genotyped as described below.

The second cohort consisted of 25 patients with bile or pancreatic duct strictures in which molecular analysis was performed on both the microdissected stained cytology cells and corresponding cytocentrifugation supernatant fluid. Fifteen of these 25 patients had known outcomes from surgical pathology examination of surgical resection specimens. In cohort 2, we aimed to assess the feasibility and utility of genotyping free DNA from cytocentrifugation supernatant fluid as produced by routine cytology practice.

Thus, between cohorts 1 and 2 we tested 33 patients with known outcomes: 18 evaluated using microdissection alone (group 1) and 15 assessed using combined stained cytology microdissection and molecular analysis of free DNA from the corresponding cytocentrifugation supernatant fluid (cohort 2A). An additional 10 patients without surgical outcomes were tested in cohort 2B.

\section{Molecular Analysis}

Mutational analysis of microdissection stained cytology slides has been previously described [7]. Briefly, we used morphologic features of cell clusters to guide the microdissection of cytology slides so as to include the cells most representative of the disease for molecular analysis. Nonetheless, some nonlesional cells were included among those microdissected and genotyped.

After cytocentrifugation of pancreaticobiliary specimens, supernatant fluid was reserved for mutational profiling. One to two milliliters of this fluid underwent DNA extraction (Qiagen) according to the manufacturer's instructions. The total storage time between cytocentrifugation and DNA extraction ranged from 3 to 8 days. The resulting DNA was resuspended in a small volume of hypotonic buffer and the concentration of extracted DNA was quantified by optical density (NanoDrop). Microdissected stained cytology cells underwent equivalent DNA extraction and resuspension. DNA amplifiability was determined by quantitative PCR targeting a 150-bp length of the first coding exon of the KRAS oncogene.

All specimens were sequenced for KRAS point mutations in codons 12 and 13 using dideoxy chain termination as previously described $[8,9]$ and further assessed for allelic imbalance/loss of 
Table 1. Cytologic and molecular features of bile duct brushings: cohort 1 (microdissected cytology slides)

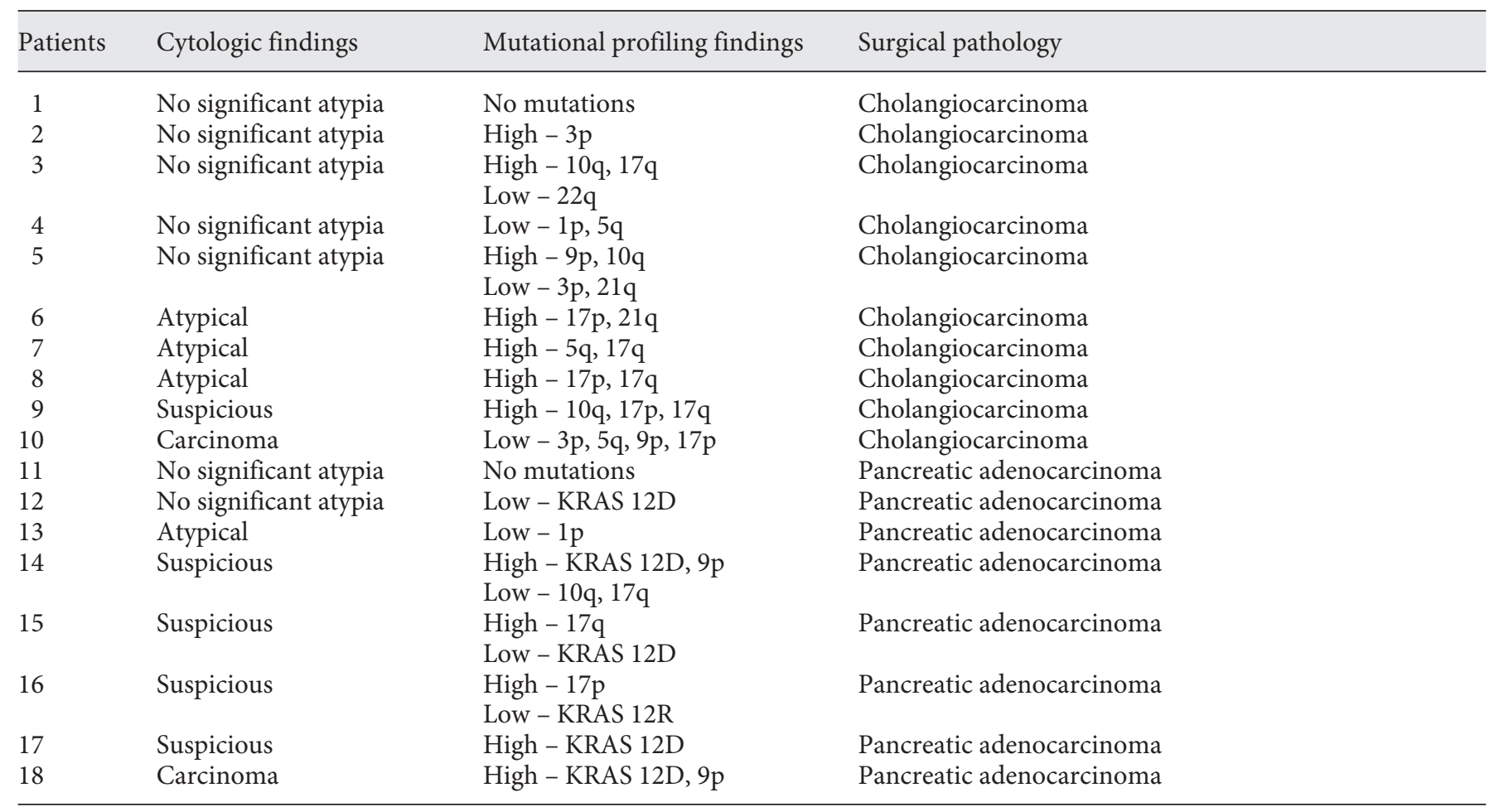

All brushings were from the bile duct. Low clonality denotes mutations that affect less than $75 \%$ of the sample DNA. High clonality indicates mutations involving $75 \%$ or more of the extracted DNA (see Methods for further details). KRAS point mutations are designated by a single letter for the substituted amino acid. All KRAS point mutations were at codon 12 of the first coding exon of the oncogene.

heterozygosity (LOH) using a panel of 16 microsatellite markers targeting common sites for tumor suppressor genes associated with pancreaticobiliary cancer. The panel contained markers at the following chromosomal locations (associated genes in parenthesis): 1p (CMM1, LMYC), 3p (VHL, OGG1), 5q (MCC, APC), 9p (CDKN2A, CDKN2B), 10q (PTEN, MXI1), 17p (TP53), 17q (NME1), and 21q, 22q (NF2) using quantitative fluorescent PCR/ capillary electrophoresis (I). The panel of markers has undergone analytic and clinical validation for pancreaticobiliary disease as reported in a number of prior studies [7-14].

Quantitative allelic imbalance determination was performed as previously described [15]. In short, the threshold for significant allelic imbalance for each microsatellite marker of the LOH marker panel was based on a large database of nonneoplastic pancreatic and bile duct specimens. This large dataset of over 1,000 specimens encompassed the majority of allele combinations seen in the general patient population available both as unfixed and fixative-treated extracted DNA. The range for normal allele balance was defined as two standard deviations from the average allele ratio in which the fluorescence derived from the shorter allele copy is divided by that of the longer allele copy. Allele ratios falling outside the thresholds were considered as demonstrating significant imbalance (LOH). When imbalance was shown to be present, an LOH clonality (degree of clonal expansion) measure- ment was approximated using the formula 1 - [(sample allelic copy ratio)/(average allele copy ratio for allele pairing) $] \times 100 \%$ when the shorter microsatellite allele copy was found to be relatively deficient. The inverse of this formula was applied when the longer allele copy was relatively deficient. For KRAS point mutation clonality assessed by dideoxy chain termination, the ratio of wild-type and mutant peak heights was used as an approximation of mutated versus nonneoplastic DNA for an individual sample. It is recognized that clonality determination for oncogene point mutation and allelic imbalance represents an approximation.

\section{Statistical Analysis}

As reported in previous work on genotyping of pancreaticobiliary strictures [7], the finding of any mutation was considered indicative of a neoplasm or cancer. This interpretation of mutations is similar to other molecular analyses of biliary strictures such as FISH analysis but is somewhat unlike our work in other neoplastic conditions in which we have used the number and clonality of mutations to characterize the degree of dysplasia present in solid tumor or cystic specimens.

The sensitivity and specificity for neoplastic process/cancer were calculated overall and then separately for microdissection and supernatant specimens. Confidence intervals for sensitivity and specificity were calculated using the exact binomial method. 


\section{Results}

A total of 43 microdissection and corresponding cytocentrifugation supernatant specimens were analyzed for DNA content and mutational profiling in two patient cohorts (tables 1,2). In 33 patients, the surgical pathology diagnosis of the resected tissue was available. These 33 samples served as control positive and negative cases to evaluate test performance. We also tested 10 cases with supernatant and cytology slides, but without surgical outcomes, to augment the comparison between molecular results from cytology slides and those from the supernatant.

Out of 33 specimens with outcome tested, mutations were detected in 25/28 malignant/neoplastic specimens, and no mutations were found in $5 / 5$ of benign/reactive specimens. The presence of any mutation was interpreted as in indication of malignancy/a neoplastic process. Using the interpretation there were no false-positive findings (i.e. mutations in surgically benign disease), and 3 false negatives, which indicates a sensitivity of $89 \%(95 \%$ CI 71-98\%) and a specificity of 100\% (95\% CI 48-100\%). Of the 10 specimens lacking outcome, mutations were identified in $1(10 \%)$ via microdissected cytology slides, and in $3(30 \%)$ via testing of supernatant fluids, showing increased sensitivity for cancer. Of note, in each of the false-negative cases (malignant specimens with no mutations) the cytologic diagnosis was also benign, suggesting that the brushing did not capture either cells or free DNA representative of the tumor.

\section{Cohort 1}

Cohort 1 consisted of 18 patients with malignant strictures where only microdissected cytology slides were genotyped - results are shown in table 1 . The cytologic diagnoses were benign (7 patients), atypical (4 patients), suspicious (5 patients), and malignant ( 2 patients), respectively. In $16 / 18$ patients of this cohort, broad panel mutational profiling revealed the presence of mutational change (sensitivity $89 \%$ ). Of note, KRAS point mutation was exclusively present in pancreatic adenocarcinoma while the spectrum of $\mathrm{LOH}$ mutational change could be seen in both pancreas and bile duct origin cancer (table 1). Two patients with confirmed cancer in which mutations could not be detected manifested benign cytology features (table 1). Both cytology and microdissectionbased mutational analysis were therefore false negative in these two patients.

\section{Cohort 2}

The results from cohort 2 , those where both microdissected cytology slides and residual supernatant fluid were genotyped, are shown in table 2. An example patient is shown in figures $1-3$. The cytology slide is shown in figure 1, and KRAS point mutation and $\mathrm{LOH}$ analysis results are shown in figures 2 and 3 for the microdissected stained cytology and corresponding cytocentrifugation supernatant fluid. Mutations were detected in 9/10 confirmed malignant specimens (sensitivity 90\%), and no mutations were detected in $5 / 5$ benign specimens (specificity $100 \%)$.

Table 3 compares the results of cytology and mutational profiling of supernatants. Mutations were detected in an additional 8/9 cases of cancer when cytology diagnosis was benign and in 5/9 cases when cases when diagnosis was atypical.

In this preliminary study, the cytocentrifugation supernatant generally yielded higher concentrations of DNA and better amplifiability of this DNA than corresponding microdissection targets (statistical analysis not needed for this small number of cases). Additionally, the DNA extracted from the supernatant fluid showed higher numbers and/or higher clonality of mutational change in 7 of 10 patients with confirmed cancers (table 2). Of the remaining 3 patients, 1 had no mutations in either cytology slides or supernatant (false negative), and in the other 2 the supernatant fluid manifested mutational change that was either fewer in number and/or of lesser clonality (patients 25 and 33; table 2).

\section{Discussion}

Molecular techniques have been successfully applied to improve sensitivity for the detection of cancer with detection of mutations serving as an efficacious ancillary tool to complement microscopic evaluation [15-18]. In particular, fluorescent in situ hybridization (FISH) has attracted significant interest as it can confer an additive benefit of $10-25 \%$ increased sensitivity for the detection of bile duct cancer [16-19]. FISH is usually performed on bile duct brushing cytology slides using a small panel of marker probes optimized for the detection of urinary bladder cancer [16-19]. The technique is robust, though in clinical practice it shares the same limitation of low cellularity and sampling variation that hinders cytology evaluation.

Others have reported on the use of PCR-based analysis for KRAS point mutation to assist the diagnosis of pancreaticobiliary strictures $[20,21]$. An approach based 
Fig. 1. Microdissection of stained cytology cells from a moderately cellular cytology slide. Microdissection was guided by the cytology features of cells most representative of the greatest degree of anaplasia (ink marks). Sufficient cellularity was judged to be present affording two separate microdissection targets for mutational analysis. Saccomanno fixation, Pap stain.

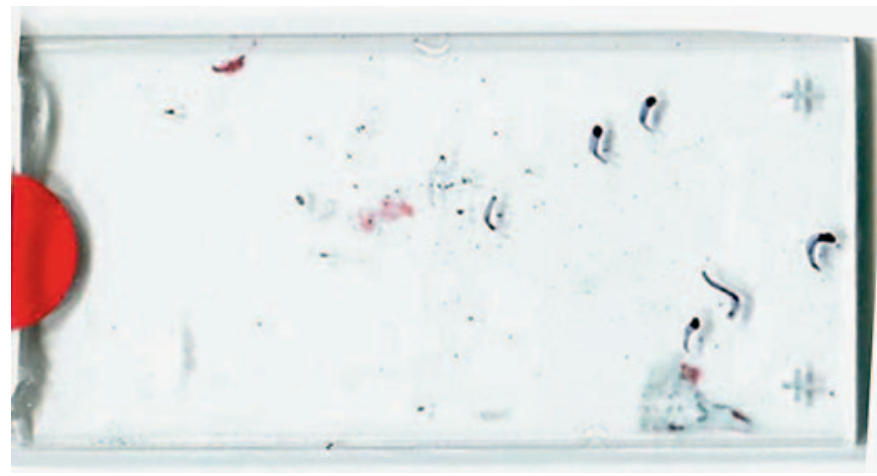

Table 2. Cytologic and molecular features of bile duct brushings: cohort 2 (microdissected cytology slides and corresponding cytocentrifugation supernatant fluid)

\begin{tabular}{|c|c|c|c|c|c|}
\hline $\begin{array}{l}\mathrm{Pa}- \\
\text { tient }\end{array}$ & $\begin{array}{l}\text { Cytologic } \\
\text { findings }\end{array}$ & $\begin{array}{l}\text { Microdissected cytology } \\
\text { mutational profile }\end{array}$ & $\begin{array}{l}\text { Supernatant fluid } \\
\text { mutational profile }\end{array}$ & $\begin{array}{l}\text { Correlation between supernatant } \\
\text { and microdissection }\end{array}$ & Surgical pathology \\
\hline 19 & No significant atypia & No mutations & No mutations & Equivalent & Reactive \\
\hline 20 & Atypical & No mutations & No mutations & Equivalent & Reactive \\
\hline 21 & Atypical & No mutations & No mutations & Equivalent & Reactive \\
\hline 22 & Atypical & No mutations & No mutations & Equivalent & Reactive \\
\hline 23 & Atypical & No mutations & No mutations & Equivalent & Reactive \\
\hline 24 & Suspicious & Low - KRAS 12V, 17q & Low - KRAS 12V, 17q & Equivalent & Cholangiocarcinoma \\
\hline 25 & Suspicious & $\begin{array}{l}\text { High }-17 p, 21 q \\
\text { Low }-3 p, 9 p, 17 q\end{array}$ & Low $-17 p, 21 q$ & Fewer mutations, lower clonality & Cholangiocarcinoma \\
\hline 26 & Carcinoma & Low $-9 p$ & High - 1p, 3p, 5q, 9p, 10q & $\begin{array}{l}\text { Higher clonality, } \\
\text { additional mutations }\end{array}$ & Cholangiocarcinoma \\
\hline 27 & Carcinoma & $\begin{array}{l}\text { High }-17 q \\
\text { Low }-10 q\end{array}$ & $\begin{array}{l}\text { High }-10 \mathrm{q}, 17 \mathrm{q} \\
\text { Low }-1 \mathrm{p}\end{array}$ & $\begin{array}{l}\text { Higher clonality, } \\
\text { additional mutations }\end{array}$ & Cholangiocarcinoma \\
\hline 28 & Suspicious & Low - KRAS 12D & Low - KRAS 12D & Equivalent & Pancreatic adenocarcinoma \\
\hline 29 & Suspicious & Low - KRAS 12D, 1p & Low - KRAS 12D, 1p, 22q & Additional mutations & Pancreatic adenocarcinoma \\
\hline 30 & Suspicious & $\begin{array}{l}\text { High - KRAS 12D } \\
\text { Low - 9p, 17p, 17q }\end{array}$ & $\begin{array}{l}\text { High - KRAS 12D, 17p } \\
\text { Low - 9p, 17q, 21q }\end{array}$ & $\begin{array}{l}\text { Higher clonality, } \\
\text { additional mutations }\end{array}$ & Pancreatic adenocarcinoma \\
\hline 31 & Carcinoma & Low - KRAS 12V, 9p & $\begin{array}{l}\text { High - KRAS 12V, 9p } \\
\text { Low - 10q, 17p }\end{array}$ & $\begin{array}{l}\text { Higher clonality, } \\
\text { additional mutations }\end{array}$ & Pancreatic adenocarcinoma \\
\hline 32 & Benign & No mutations & No mutations & Equivalent & Ampullary carcinoma \\
\hline 33 & Atypical & Low $-10 q, 17 p, 17 q$ & Low $-17 p$ & Fewer mutations & Hepatocellular carcinoma \\
\hline 34 & No significant atypia & No mutations & No mutations & Equivalent & Not available \\
\hline 35 & No significant atypia & No mutations & No mutations & Equivalent & Not available \\
\hline 36 & No significant atypia & No mutations & No mutations & Equivalent & Not available \\
\hline 37 & No significant atypia & No mutations & No mutations & Equivalent & Not available \\
\hline 38 & Atypical & No amplification of KRAS or LOH & No mutations & Better amplification & Not available \\
\hline 39 & Atypical & No mutations & No mutations & Equivalent & Not available \\
\hline 40 & Atypical & Low - KRAS 12D & Low - KRAS 12D, 9p, 17p & Additional mutations & Not available \\
\hline 41 & Suspicious & No mutations & No mutations & Equivalent & Not available \\
\hline 42 & Suspicious & No mutations & Low $-1 \mathrm{p}$ & Additional mutations & Not available \\
\hline 43 & Suspicious & No mutations & Low $-1 \mathrm{p}$ & Additional mutations & Not available \\
\hline
\end{tabular}

Specimens assessed as hypocellular or acellular were categorized as with 'no significant atypia'. All brushings were from the bile duct except for patient samples 30 and 42 . Low clonality denotes mutations that affect less than $75 \%$ of the sample DNA. High clonality indicates mutations involving $75 \%$ or greater of the extracted DNA (see Methods for further details). KRAS point mutations are designated by a single letter for the substituted amino acid. All KRAS point mutations were at codon 12 of the first coding exon of the oncogene. 


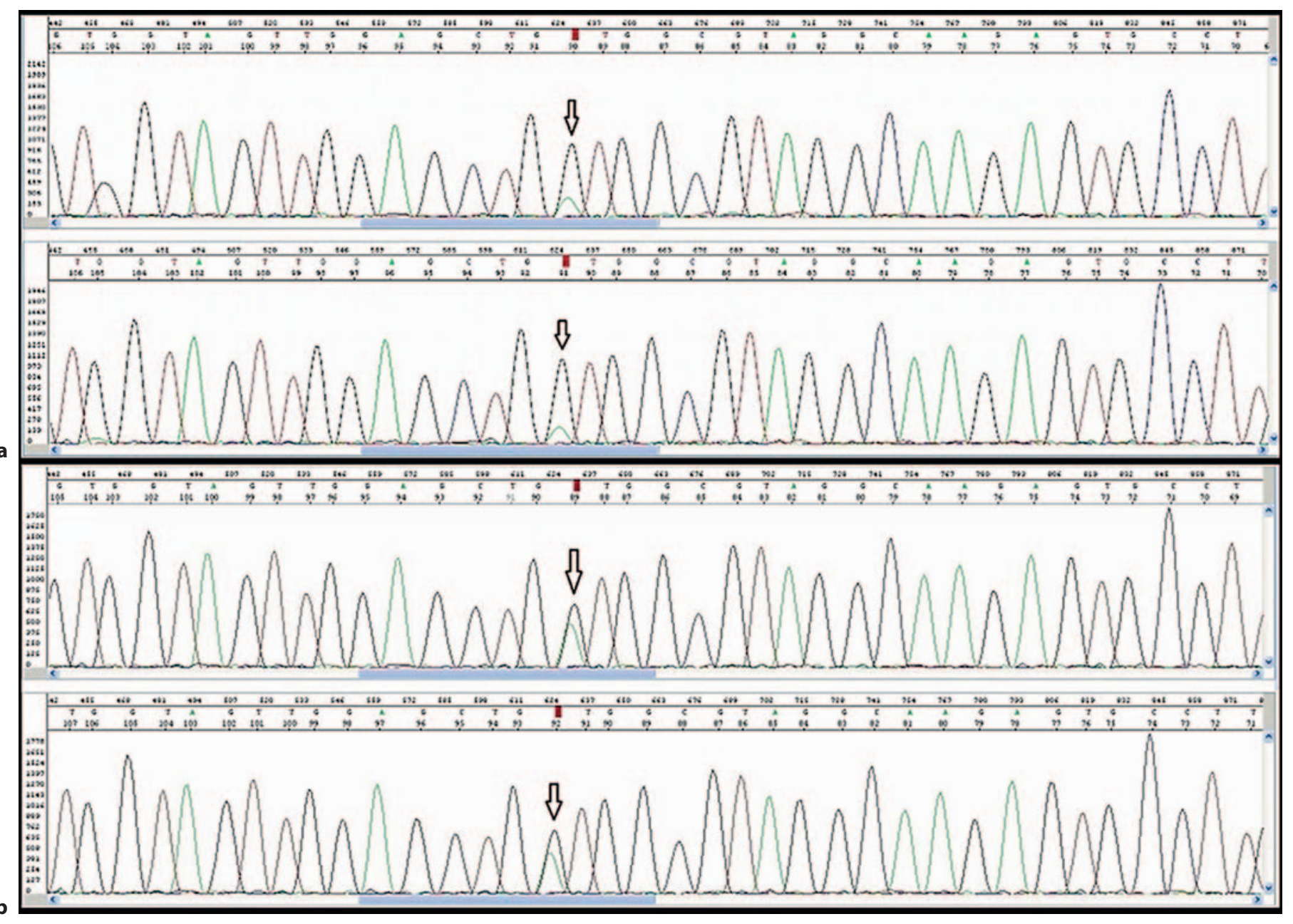

Fig. 2. KRAS point mutational analysis of microdissected stained cytology (a) and cytocentrifugation supernatant fluid (b). Both sample types show the presence of the same KRAS point mutation (codon 12 substitution of the normal glycine by valine) in the majority of cellular DNA (high clonality KRAS point mutation).

strictly on examination of KRAS has two significant shortcomings. The first is the low frequency of KRAS point mutation in bile duct cancer from patients in the Western hemisphere [22], resulting in low sensitivity for primary cholangiocarcinomas. The second shortcoming is the inability to differentiate between benign versus malignant pancreatic ductal processes using KRAS point mutation detection alone since acquisition of this oncogene mutation occurs in both benign and malignant neoplastic ductal lesions [20,21]. There continues to be a need for ancillary testing that does not compete for precious cellular material derived from pancreatic and bile duct stricture sampling yet is robust and effective on the paucicellular and acellular samples frequently encountered in the workup of patients.
While pancreatic and bile duct cancers share many mutational events such as tumor suppressor mutation and loss involving p53, CDKN2A, and PTEN [22], these two forms of cancer possess notable differences. First, KRAS point mutation is present in approximately $85 \%$ of pancreatic cancer while it occurs infrequently in bile duct cancer [22]. Also certain forms of tumor suppressor gene damage such as that affecting DPC4 are characteristic of pancreatic cancer but not of cholangiocarcinoma. Since both of these cancers can produce distal common bile duct and pancreatic duct obstruction, it is reasonable to seek a molecular approach encompassing markers that address both of these malignancies.

Previous work has demonstrated the utility of ancillary molecular testing using microdissection of cells from un- 


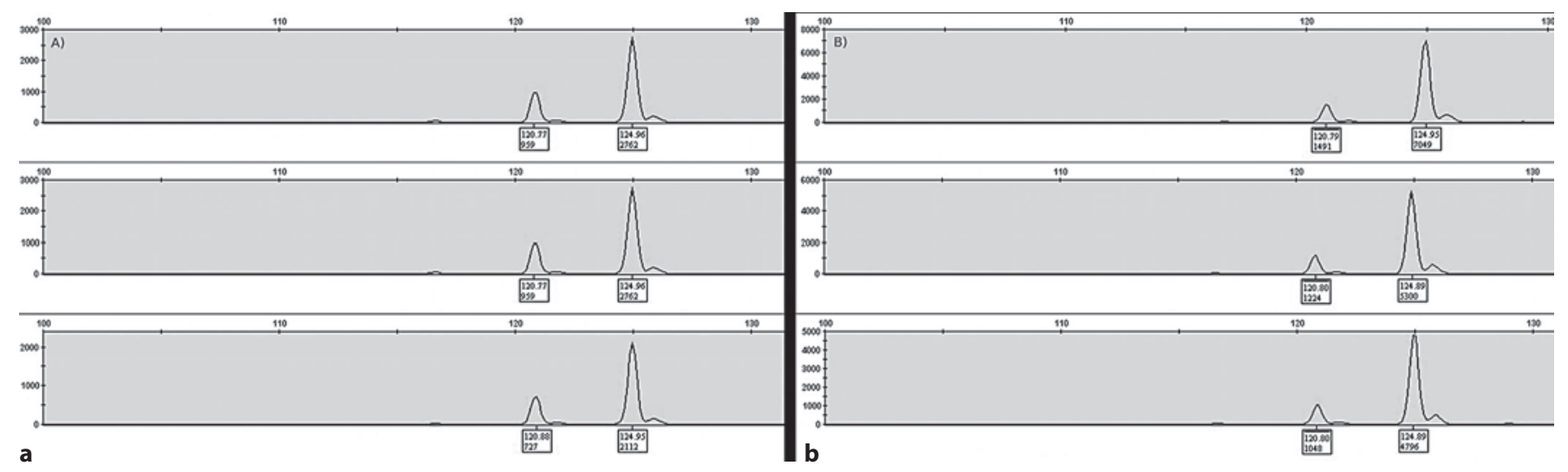

Fig. 3. Allelic imbalance analysis of microdissected stained cytology (a) and cytocentrifugation supernatant fluid (b) performed in triplicate. Both sample types show the presence of the same LOH mutation; however, the imbalance is greater in the cytocentrifugation supernatant fluid sample (high clonality LOH mutation) compared to the microdissected stained cytology cell DNA (low clonality LOH mutation).

stained recut cell block tissue sections or stained cytology slides [7]. By relying on polymerase chain reaction (PCR) to amplify small amounts of representative DNA, microdissection of targeted cells can resolve an indeterminate microscopic diagnosis as well as provide clinically actionable information [7]. As in the case of FISH, a drawback of the microdissection approach is the need for an adequate number of representative cells (although microdissection-based mutational profiling requires relatively low numbers of cells). Moreover, in microdissection, the representative cells must be isolated from nonneoplastic supporting cells (mesenchymal stromal cells, inflammatory cells), as inclusion of DNA from the nonneoplastic cells can mask the detection of mutations in affected cells. Furthermore, when specimen cellularity is low, there can be a reluctance to utilize limiting numbers of stained cytology cells for any ancillary molecular analysis (e.g. FISH or microdissection-based mutational profiling), especially when the most representative cells are confined to a single glass slide. For these reasons, we sought alternative ways to increase the yield of potentially mutated DNA information from these problematic specimens.

We have shown, in a small cohort of pancreaticobiliary brush specimens, that it is possible to detect mutational change both in microdissected stained cells removed from cytology slides and in the free DNA present in the cytocentrifugation supernatant fluid (table 2). Most notably, the free DNA content of supernatant proved to be superior to the microdissected stained cytology slides to search for and characterize mutational change, especially when the overall cellularity was low. This supernatant
Table 3. Comparison of cytologic assessment, supernatant mutational profiling, and surgical pathology outcome

\begin{tabular}{lllll}
\hline \multirow{2}{*}{ Cytologic findings } & $\begin{array}{l}\text { Supernatant } \\
\text { mutation } \\
\text { profiling }\end{array}$ & \multicolumn{2}{l}{ Outcome } & \multirow{2}{*}{ Total } \\
\cline { 3 - 4 } & benign & cancer & \\
\hline No significant atypia & Negative & 1 & 3 & 4 \\
& Positive & & 5 & 5 \\
\hline Atypical & Negative & 4 & 5 & 4 \\
\hline Suspicious & Positive & & 10 & 10 \\
\hline Carcinoma & Positive & & 5 & 5 \\
\hline Total & Positive & & 5 & 33 \\
\hline
\end{tabular}

fluid is currently discarded, yet its performance characteristics are consistent with it being a source of enriched DNA from the most actively proliferating cells; hence it is an excellent source of neoplastic DNA when cancer is under investigation. Supernatant fluid analysis affords a noncompeting way to characterize molecular changes that in turn can contribute valuable information differentiating reactive from neoplastic cell proliferation. In this series, mutational profiling was most helpful when cytology diagnoses were benign or atypical - mutations were detected in an additional 8/9 cases of cancer when the cytology diagnosis was benign and in $5 / 9$ cases when cases when the diagnosis was atypical.

Comparing the two sources of DNA, microdissected stained cytology cells and cytocentrifuged supernatant 
fluid, the detectable mutational change was equal to or greater in the supernatant specimens, suggesting that the supernatant may be enriched with DNA from the most actively proliferating neoplastic cells in the nearby tumor. We were surprised that the supernatant fluid would be superior to the microdissected stained cytology for molecular analysis since the supernatant, by definition, was acellular and expected to yield little if any amplifiable DNA, but indeed the supernatant most frequently provided better results than the microdissected cytology slide. While in vitro DNA degradation effects related to fixative exposure, specimen handling and cell staining may diminish the amplifiability of microdissected stained cytology cells, even in paucicellular samples the supernatant fluid yielded distinctly higher amounts and more intact DNA compared to microdissected cells. This is notably similar to findings regarding circulating free DNA and suggests that mutation-enriched free DNA is likely to be present in cytocentrifugation supernatants from a wide variety of tumor types [5].

Combining microdissection and supernatant fluid molecular analysis is especially useful as each approach offers unique advantages without competing with the others while complementing microscopic examination. Cytology examination provides insights that can guide microdissection to selectively remove the most altered cells to serve as a substrate for detailed mutational analysis. When atypia is seen, the presence of mutations affirms that it is neoplastic rather than reactive in origin. The corresponding supernatant fluid can be used to confirm the presence of mutational change derived from these cells or interrogate cells in the vicinity of the stricture, but not captured by the cytobrush. In this way molecular analysis of supernatant fluid may be particularly helpful in addressing sampling variation.

We recognize several limitations of this study. First, the total number of test samples is relatively small and the results shown here require confirmation with additional specimens. In particular, the addition of more confirmed negative specimens would strengthen the findings around sensitivity. Second, this study was restricted to the use of Saccomanno's fixation which, though commonly used, is not the only fixative used in cytology practice. While each fixative merits individual testing with respect to its capacity to deliver adequate levels of representative, intact supernatant DNA for mutational profiling, it is reasonable to expect favorable results with other methods of sample preparation as most cytology fixatives are alcohol based and not expected to induce significant DNA degradation. Indeed, additional unpublished work in our lab involving testing of other supernatant fluids indicates that most common cytology fixatives yielded amplifiable DNA (with the notable exception of CytoRich Red). This is consistent with our prior experience in genotyping microdissected cytology slides, in which most slides yielded amplifiable DNA regardless of the cytology fixative used. While none of the supernatant specimens evaluated here failed to provide adequate DNA for mutational profiling, it is expected that a small proportion of markedly hypocellular specimens, likely from nonneoplastic states, will fail to meet the lower limit of DNA quantity for analysis.

It should be noted that in this study, when microdissection alone was used, $2 / 18$ cases proved to be false negative for mutation detection (1 cholangiocarcinoma and 1 pancreatic adenocarcinoma; table 1 ). While no falsenegative malignant stricture cases were seen in cohort $2 \mathrm{~A}$ where both microdissection and supernatant fluid analysis were utilized, in two patients, the supernatant fluid manifested a lesser extent of mutational change than that present in the corresponding microdissected stained $\mathrm{cy}$ tology cells. These findings emphasize that the sampling variation and other limitations may nevertheless be present in individual cases limiting or preventing the detection of cancer. It remains essential to integrate all of the information including clinical and imaging findings to optimize individual patient diagnosis.

It can be important to consider whether the changes seen in the supernatant fluid could simply result from the breakdown of cells during the centrifugation step of specimen handling. This in turns goes to the issue of whether analysis of the supernatant fluid reflects the status of the extracellular fluid space or whether it is a surrogate for the cells within the aspirated sample. Cytocentrifugation is generally carried out at speeds below which there is significant cell disruption. More noteworthy, with regard to cell disruption, are the findings of cases wherein the microdissected cells failed to manifest detectable mutations present in the supernatant fluid. It is therefore unlikely that cell disruption could account for the differences in mutational profiles here. Rather the supernatant fluid behaves more as a reservoir of mutated free DNA derived from cells included in the aspiration or from cells in the vicinity of brushing.

There is evidence in this initial study that hypocellular cytology specimens assessed as inadequate for microscopic examination can reliably provide analyzable supernatant fluid DNA and as such can be expected to improve the sensitivity of detection of neoplastic disease when used in an ancillary testing role. More studies are underway to fur- 
ther assess the clinical value of the molecular information from cytocentrifugation specimens in this regard.

One of the greatest challenges for the early diagnosis of cancer is sampling variation in light of the topographic tissue heterogeneity that is fundamental to solid organ neoplasia. This is particularly true of the cytobrush sampling where neoplastic disease can be missed if the sample procurement does not capture the most advanced neoplastic cells. Sampling variation can occur when the most advanced stage of neoplastic disease development is missed or when inflammation or stromal cells are included during the aspiration process. To address this especially important problem, we searched for mutational change in free DNA released from neoplastic cells into their extracellular environment.

\section{Conclusion}

In this study we have shown that neoplastic free DNA is present in the extracellular compartment even when a particular cytology sample lacks sufficient cellularity to afford a definitive diagnosis. Most importantly, the cellfree supernatant, available as a residual specimen after cytocentrifugation, should be regarded as a potentially valuable source of information due to its content of adequate amounts of free DNA for robust mutational analysis with the capacity to address issues related to sampling variation and to detect neoplasia at an early stage of development.

\section{References}

1 Anupindi SA, Victoria T: Magnetic resonance cholangiopancreatography: techniques and applications. Magn Reson Imaging Clin N Am 2008; 16:453-466.

$\checkmark 2$ Coté GA, Sherman S: Advances in pancreatobiliary endoscopy. Curr Opin Gastroenterol 2010;26:429-435.

-3 Siddiqui AA, Kowalski TE, Shahid H, O'Donnell S, Tolin J, Loren DE, Infantolino A, Hong SK, Eloubeidi MA: False-positive EUS-guided FNA cytology for solid pancreatic lesions. Gastrointest Endosc 2011;74: 535-540.

4 Rogart JN, Loren DE, Singu BS, Kowalski TE: Cyst wall puncture and aspiration during EUS-guided fine needle aspiration may increase the diagnostic yield of mucinous cysts of the pancreas. J Clin Gastroenterol 2011;45:164-169.

5 Aung KL, Board RE, Ellison G, Donald E, Ward T, Clack G, Ranson M, Hughes A, Newman W, Dive C: Current status and future potential of somatic mutation testing from circulating free DNA in patients with solid tumours. Hugo J 2010;4:11-21.

6 Bibbo M: How technology is reshaping the practice of nongynecologic cytology: frontiers of cytology symposium. Acta Cytol 2007;51:123-152.

7 Khalid A, Pal R, Sasatomi E, Swalsky P, Slivka A, Whitcomb D, Finkelstein S: Use of microsatellite marker loss of heterozygosity in accurate diagnosis of pancreaticobiliary malignancy from brush cytology samples. Gut 2004;53:1860-1865.

8 Gamblin TC, Finkelstein SD, Upsal N, Kaye JD, Blumberg D: Microdissection-based allelotyping: a novel technique to determine the temporal sequence and biological aggressiveness of colorectal cancer. Am Surg 2006;72:445-453.
-9 Schoedel KE, Finkelstein SD, Ohori NP: KRas and microsatellite marker analysis of fine-needle aspirates from intraductal papillary mucinous neoplasms of the pancreas. Diagn Cytopathol 2006;34:605-608.

10 Lin X, Finkelstein SD, Zhu B, Ujevich BJ, Silverman JF: Loss of heterozygosities in Barrett esophagus, dysplasia, and adenocarcinoma detected by esophageal brushing cytology and gastroesophageal biopsy. Cancer 2009;117:57-66.

11 Lapkus O, Gologan O, Liu Y, Swalsky PA, Wilson MM, Finkelstein SD, Silverman JF: Determination of sequential mutation accumulation in pancreas and bile duct brushing cytology. Mod Pathol 2006;19:907-913.

12 Krishnamurti U, Sasatomi E, Swalsky PA, Finkelstein SD, Ohori NP: Analysis of loss of heterozygosity in atypical and negative bile duct brushing cytology specimens with malignant outcome: are 'false-negative' cytologic findings a representation of morphologically subtle molecular alterations? Arch Pathol Lab Med 2007;131:74-80.

13 Khalid A, Zahid M, Finkelstein SD, LeBlanc JK, Kaushik N, Ahmad N, Brugge WR, Edmundowicz SA, Hawes RH, McGrath KM: Pancreatic cyst fluid DNA analysis in evaluating pancreatic cysts: a report of the PANDA study. Gastrointest Endosc 2009; 69:1095-1102.

14 Toll AD, Kowalski T, Loren D, Bibbo M: The added value of molecular testing in small pancreatic cysts. JOP 2010;11:582-586.

15 Mohan D, Finkelstein SD, Swalsky PA, Sasatomi E, Wiley C, Hamilton RL, Lieberman F, Couce ME: Microdissection genotyping of gliomas: therapeutic and prognostic considerations. Mod Pathol 2004;17:1346-1358.
16 Kipp BR, Stadheim LM, Halling SA, Pochron NL, Harmsen S, Nagorney DM, Sebo TJ, Therneau TM, Gores GJ, de Groen PC, Baron TH, Levy MJ, Halling KC, Roberts LR: A comparison of routine cytology and fluorescence in situ hybridization for the detection of malignant bile duct strictures. Am J Gastroenterol 2004;99:1675-1681.

17 Levy MJ, Baron TH, Clayton AC, Enders FB, Gostout CJ, Halling KC, Kipp BR, Petersen BT, Roberts LR, Rumalla A, Sebo TJ, Topazian MD, Wiersema MJ, Gores GJ: Prospective evaluation of advanced molecular markers and imaging techniques in patients with indeterminate bile duct strictures. Am J Gastroenterol 2008;103:1263-1273.

18 Barr Fritcher EG, Caudill JL, Blue JE, Djuric K, Feipel L, Maritim BK, Ragheb AA, Halling KC, Henry MR, Clayton AC: Identification of malignant cytologic criteria in pancreatobiliary brushings with corresponding positive fluorescence in situ hybridization results. Am J Clin Pathol 2011;136:442-449.

19 Smoczynski M, Jablonska A, Matyskiel A, Lakomy J, Dubowik M, Marek I, Biernat W, Limon J: Routine brush cytology and fluorescence in situ hybridization for assessment of pancreatobiliary strictures. Gastrointest Endosc 2012;75:65-73.

20 Rashid A: Cellular and molecular biology of biliary tract cancers. Surg Oncol Clin N Am 2002;11:995-1009.

21 Nault JC, Zucman-Rossi J: Genetics of hepatobiliary carcinogenesis. Semin Liver Dis 2011;31:173-187.

22 Fasanella KE, Krasinskas A, Schoedel KE, Sasatomi E, Slivka A, Whitcomb DC, Sanders M, Nodit L, Raab S, McGrath KM, Ohori NP, Khalid A: DNA mutational differences in cytological specimens from pancreatic cancer and cholangiocarcinoma. Pancreatology 2010;10:429-433. 